\title{
IIUM Bus On Campus Monitoring System
}

\author{
Muhammad Nur Zaki Juhari and Hasmah Mansor \\ Department of Electrical and Electronic Engineering \\ Kulliyyah of Engineering, International Islamic University Malaysia \\ 53100 Gombak, Kuala Lumpur, Malaysia \\ mnzaki10@gmail.com, hasmahm@iium.edu.my
}

\begin{abstract}
Malaysia has invested on public transport over the years to improve quality of leaving. Transportation has been identified as the key area in Malaysia's government transformation programme. In Malaysia, many people still choose to use their own transport compared to public transport to travel. For efficient public transport service such as bus, real-time tracking/monitoring must be available apart from their fixed schedule. The objective of this project is to develop a low cost real-time IIUM bus on campus monitoring system. IIUM shuttle bus monitoring has been chosen as a pilot study which reflects the bigger picture of Malaysia's bus system. In IIUM, students need to wait for the bus without any clue and this is such a waste of time. Thus, IIUM bus tracking system is proposed in this project where waiting time can be improved. Users can track the exact location of the bus therefore they can have better plan for their journey. Global positioning system technology has been used to send and receive the coordinate data to the server. A web server application has been developed to integrate the Global positioning system data to be presented on the map. At the end of the project, a website of real-time IIUM bus on campus monitoring system has been developed where users can monitor the exact location of the IIUM bus conveniently.
\end{abstract}

Keywords - GPS tracker; vehicle monitoring; web server; Arduino microcontroller.

\section{INTRODUCTION}

Malaysia's National Key Result Area (NKRA) 2014 under Government Transformation Programme (GTP) was identified to improve the socio economy growth of Malaysian. One of the seven major sectors of NKRA is the improvement of urban public transport. Malaysia has invested on public transport over the years however there are still more people choose to use their own transport to travel hence incurrence of massive traffic. There are many reasons related this issue, one of which is inaccurate and inefficient public transport schedule. For example, bus service, bus is not popular in Malaysia due to the unavailability of centralized bus tracking or monitoring.

There are many researches those have been conducted related to tracking or monitoring. The most common technology for tracking is Global Positioning System (GPS). GPS can be used to track, navigate and locate. GPS can be found in airplanes, cars, smartphones, boat and others. Zuki has developed Vehicle Tracking System by enhancing GSM with GPS [1]. The project involved GPS receiver and microcontroller, integration between GSM module and microcontroller and integration between sensor and microcontroller. The proposed vehicle tracking system has additional feature of security system that allows users to monitor their vehicle remotely. Similarly, Lu and Chen have utilized microcontroller, external memory and oscillator to design Mobile Tracking System. The system is designed to work as a position locator to track expensive property or important cargo. The user is able to request position data by calling the system, built around Intel $80 \times 51$ microcontroller using cellular phone or an ordinary phone [2]. Flores (2013) has used GPS coupled with inertia measurement unit (IMU) for visual impaired people. The proposed navigation system gives the information of heading and distance to the final destination to the user. The system was built on the Android smart phone and the connection to IMU is via Bluetooth [3]. Mistary and Chile (2015) have developed real-time vehicle tracking using GPS and GSM technologies. The graphical user interface (GUI) was developed by using Matlab and then interfaced with Google earth [4]. Test results showed that the vehicle was tracked successfully, form one point to another point in the university compound. The GUI can only observed from local computer. It was part of their future plan to develop a web based tracking system and mobile based messaging application.

In case of public transport, for example bus usually has fixed time schedule for every bus stop. However, traffic conditions, bus driver's factor and weather may cause delay in the service. Tracking of bus public transport using GPS and view from Google Maps is a good alternative, as proposed in this project. The web server based application will receive the GPS data from the server in order to be visualised the tracking of bus location in the dedicated route. By having this system, people can manage their time more efficiently.

\section{HOW GPS WORKS FOR TRACKING SYSTEM}

There are two elements need to be identified in GPS tracking which are the satellites location and the distance from the device. GPS will transmit data signal while orbiting the earth twice a day while GPS receiver stores and controls the coded information data that received from the satellites. The GPS data contains the approximate positions of the satellites those orbiting the earth. 
The position of the user (or referred to bus in this project) can be determined by measuring the distance from satellites. Fig. 1 shows the $2 \mathrm{D}$ position (latitude and longitude) and track movement of the user/bus can be determined when the GPS receiver receives signal from at least three satellites.

Meanwhile, in Fig. 2 the 3D position of the user (latitude, longitude and altitude) can also be computed if the GPS receiver receives signal from four or more satellites [4]. More accurate position can be obtained if GPS receiver receives signals from more satellites. The other user's information such as bearing, speed, sunrise time, and track can be calculated as well [6].

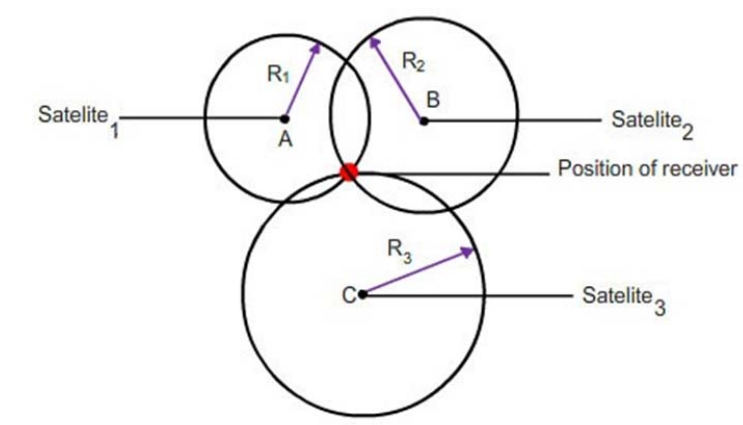

Figure 1. 2D user position [5]

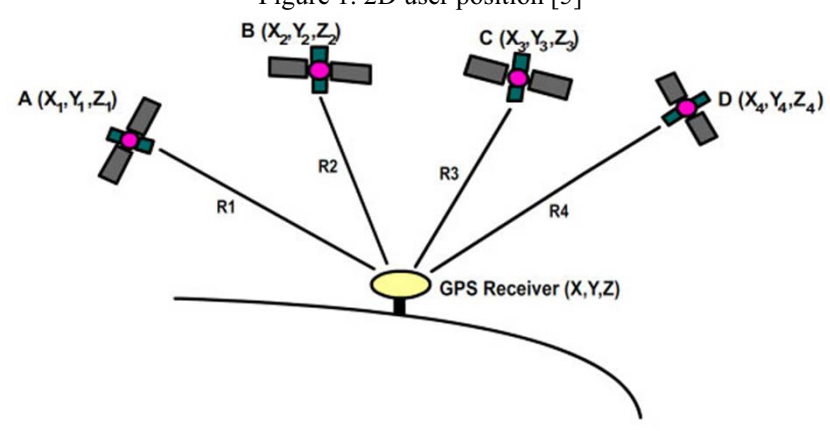

Figure 2. 3D user position

In this project, web server has been developed and integrated with the GPS data. The web server is the medium for the user to identify the exact location of the bus. More detail info about webserver can be found from [7].

Other tools that have been used to develop IIUM bus monitoring system are AWS Amazon Cloud Server and Google mas API V3. Amazon Elastic Compute Cloud (EC2) forms a central part of Amazon.com's cloudcomputing platform, Amazon Web Services (AWS), by allowing users to rent virtual computers on which to run their own computer applications. EC2 encourages scalable deployment of applications by providing a web service through which a user can boot an Amazon Machine Image to configure a virtual machine, which Amazon calls an "instance", containing any software desired. A user can create, launch, and terminate server-instances as needed, paying by the hour for active servers - hence the term "elastic". EC2 provides users with control over the geographical location of instances that allows for latency optimization and high levels of redundancy [8].

Google Maps Tracks API v3 allows developers to build apps that can store, display and analyze GPS data on a map. This API allows organizations to develop customized location-based applications to meet specific business needs [9].

\section{METHODOLOGY}

\section{A. General Block Diagram}

Tracking moving object is an exciting field that involves in the use of GPS receiver. The project includes integration of web server and a GPS receiver and should be able to display the location of the shuttle bus on the Google Maps of the website. As shown in Fig. 3 the project is divided into three parts; hardware, server or database and website.

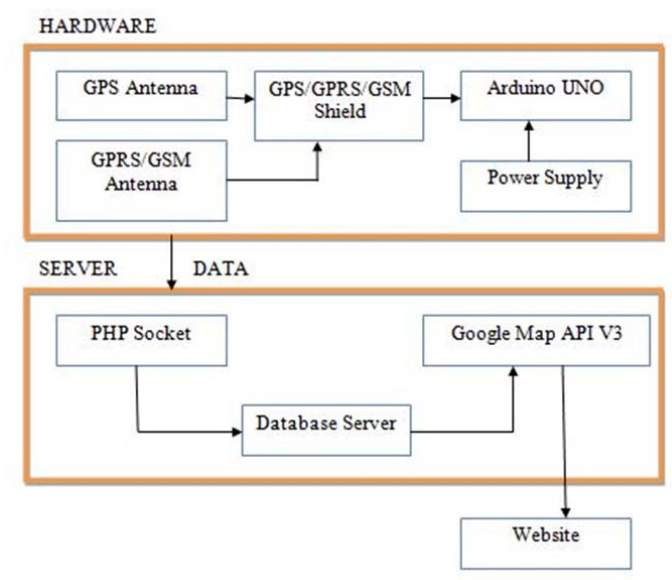

Figure 3. General Block Diagram

Generally, GPS receiver is used to acquire the location data of the bus. The location data is sent to the PHP file in the server by sending HTTP Get Request. The PHP processes the data and sends to the database to be stored. The web page takes the data from the database and display on the map. The web server takes the data form the database and displays the location of the device. The map is obtained from the Google Services (Google Maps API v3).

\section{B. Hardware Description}

\section{1) Arduino UNO}

Arduino Uno is a board based on the ATmega328 microcontroller. It has 14 digital input/output pins (of which 6 can be used as PWM outputs), 6 analog inputs, a $16 \mathrm{MHz}$ ceramic resonator, a USB connection, a power jack, an ICSP header, and a reset button [9]. GPS shield has been added to the Arduino Uno. Arduino has been used as the controller of the process involving GPS receiver and GSM/GPRS module. 


\section{2) $G P S / G P R S / G S M$ Module V3.0}

GPS/GPRS/GSM Module supports GPS technology for satellite navigation. It is possible for the control system to send messages and use the GSM network.

It is controlled via AT commands (GSM07.07,07.05 and SIMCOM enhanced AT Commands). The design of this shield allows user to drive the GSM \& GPS function directly with the computer and the Arduino board. It includes a high-gain SMD antenna for GPS \& GSM. The GPS/GPRS/GSM shield uses an embedded SIM908 chip from SIMCom (Fig. 4).

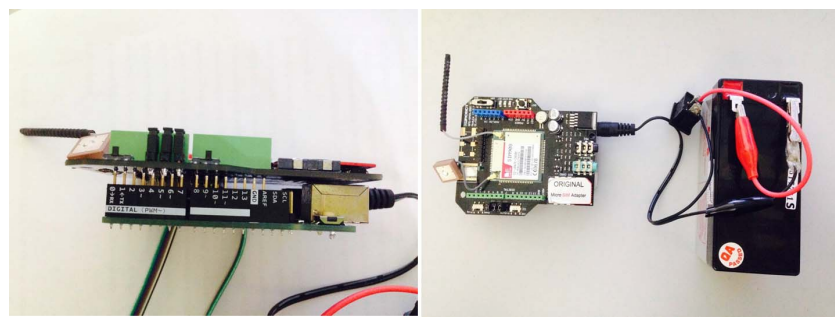

Figure 4. GPS/GPRS/GSM Module with 9V power supply

The Arduino sketch coding is uploaded into the microcontroller. SIM card used is provided from Celcom telco operator. The Access Point Name (APN) is configured on the Arduino sketch coding to enable the GPRS network to connect with the operator service. The web server URL also needs to be included in the sketch coding to send the data to the server. Sample of code for Access point name and send data through URL are shown in Fig. 5 and Fig. 6 respectively.

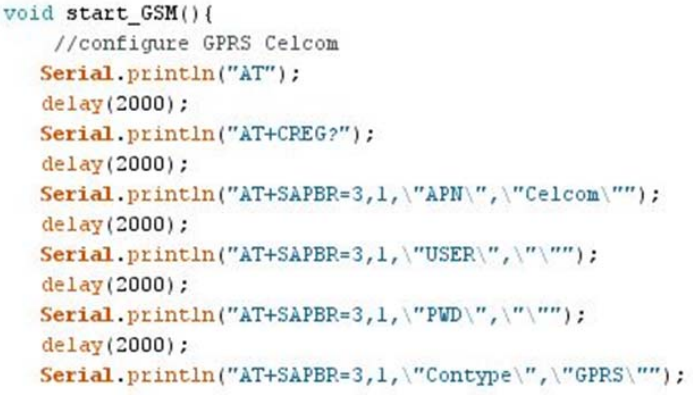

Figure 6. Send data through 'URL'

During the setup, there are several precautions to be taken to avoid connections failure:

- The GPS antenna must be in horizontal position.

- SIM card should have enough credit to connect with internet.
- Check if the external battery voltage is low, the shield needs extra power.

- Check signal range, best to be on an area with full coverage.

- $\quad$ Signal for GPS has best performance on a clear direct line of sight with sky, even better with less building around.

- GPS needs time to connect to at least 4 satellites to output data.

\section{Software}

When the GPS fix the GPS satellites, the GPRS/GPS shield will connect to the network and it will send the GPS data through the Internet to the PHP script in the server computer. Software system must be setup to display the location on the web server.

\section{1) Setup Ubuntu Server}

To enable the data receive from the internet, a cloud server is needed. Ubuntu server is one of the free servers that can be used. To use the web server service, first, registration and configuration need to be done according to the project specifications. In this project HTTP has been used to transport the data from the GPS to the server via GPRS internet. Fig. 7 and Fig. 8 show AWS dashboard and the login process in Ubuntu server. Server is important in order to save and read the data from the GPS.

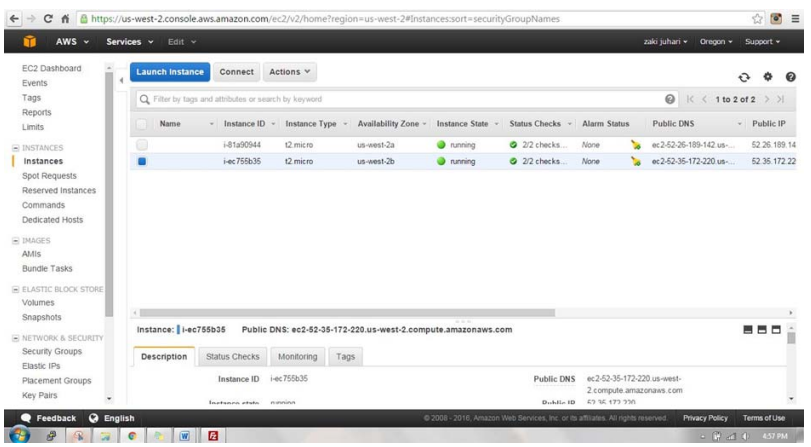

Figure 7. AWS dashboard

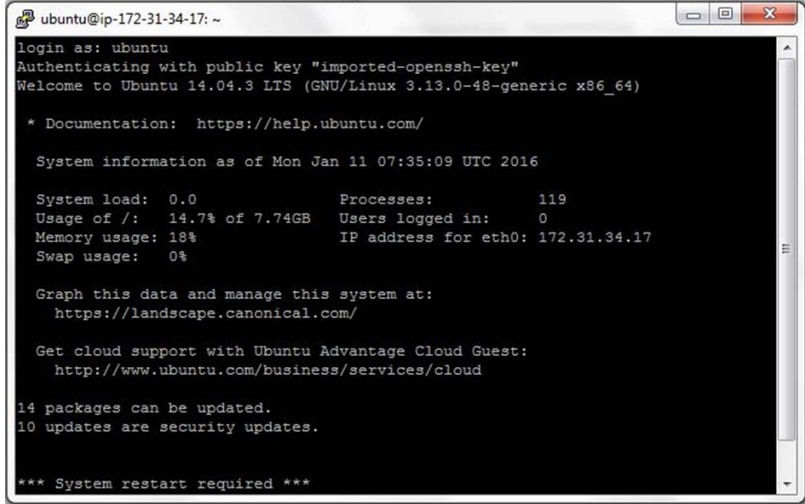

Figure 8. Log in Ubuntu Server to Setup PHP, Apache, MySQL 


\section{2) PHP Script}

By using 'GET' method in PHP script to call the data from the GPS/GPRS shield (Fig. 9), the GPS data will save in the Ubuntu server installed with PHP, Apache, and MySQL. To include the Google Maps in the web server, Google API V3 key must be set in the PHP script. This will make the GPS data available on map for visualization. FileZilla Software is needed to deploy the PHP script (Fig. 10).

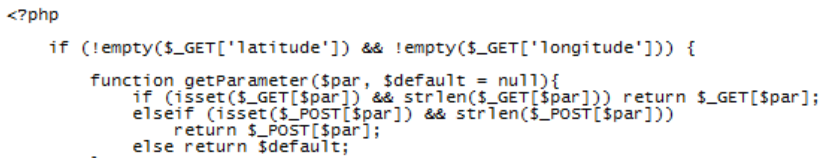

Figure 9. Using Get Method to retrieve GPS data

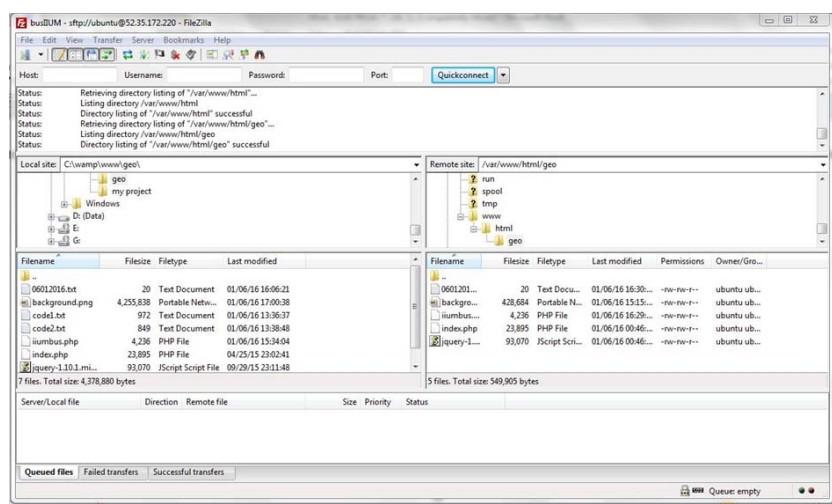

Figure 10. FileZilla Software to deploy PHP script

\section{RESULT AND ANALYSIS}

\section{A. Testing the GPS receiver}

In the preliminary test, GPS receiver has been verified to be function and in good conditions. Precautions have been taken in order to get the GPS coordinates.

- Check external battery power levels, the shield needs extra power. The power need is $5 \mathrm{~V}$ to power on the whole system.

- Check signal range, best to be on an area with full coverage.

- Signal for GPS has best performance on a clear direct line of sight with sky such as outdoor.

- GPS needs time to connect to at least 4 satellites to output data. From the experiment, about 10 minutes for the duration of the GPS to receive the coordinate data of the GPS receiver for the first time.

\section{B. Output Data From Serial Monitor}

The experiment has been conducted in two different areas; near to buildings and outdoor (line of sight with sky). In the first test (near to building), the result is shown in Fig. 11. Serial monitor output shows 0 coordinates for both latitude and latitude that indicates no GPS data received from the satellite.
Fig. 12 shows the outputs from the serial monitor when the GPS receiver was tested outdoor with open sky. When the receiver connected to at least four satellites, the coordinate data is received. Then the coordinate is sent to the server. The coordinate of the receiver is determined by calculating the distance from 4 satellites in 3-dimension. The distance between the receiver and GPS satellites can be determined by estimating the amount of time taken by each signals to reach the receiver. The signals are moving at the speed of light.

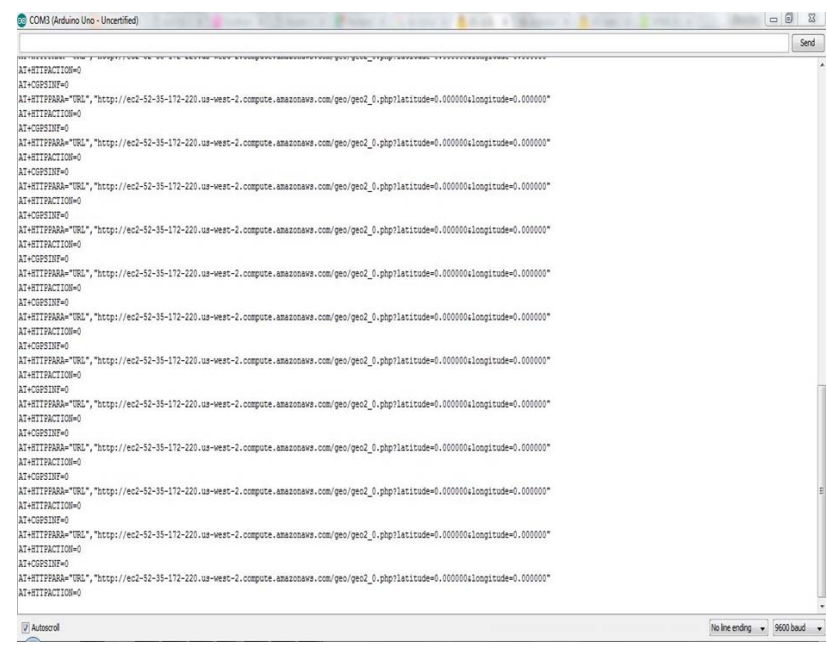

Figure 11. GPS data from serial monitor - tested near to buildings

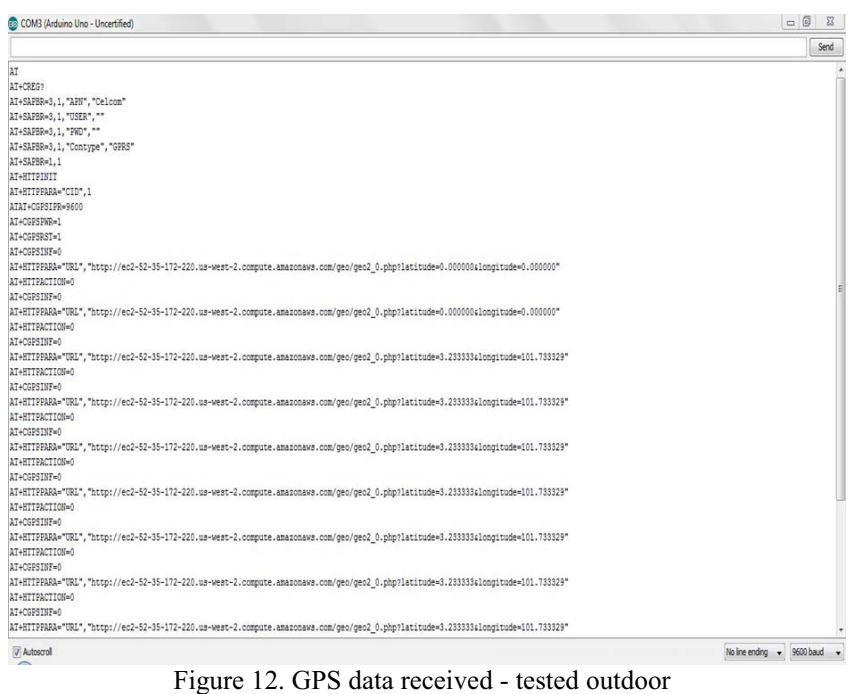

GPS Accuracy depends on a number of variables, most notably signal to noise ratio (noisy reception), satellite position, weather and obstructions such as buildings and mountains. These factors can create errors in your perceived location. Signal noise usually creates an error from around one to ten meters. Mountains, buildings and other things that might obstruct the path between the receiver and the satellite can cause three times as much error as signal noise. A GPS receiver must be able to get a lock on 4 satellites to be able to solve for a position. Besides that, the gain is the 
efficiency of the antenna in any given orientation. This applies to both transmitting antennas and receiving antennas. In this project we are using high gain GPS and GSM antenna.

\section{Web server}

After PHP script has been deployed. Fig. 13 shows the website that contains Google Maps as the monitoring interface for this project. IIUM campus is set as the centre of the map, including bus stop icon and arrow showing the route IIUM bus shuttle.

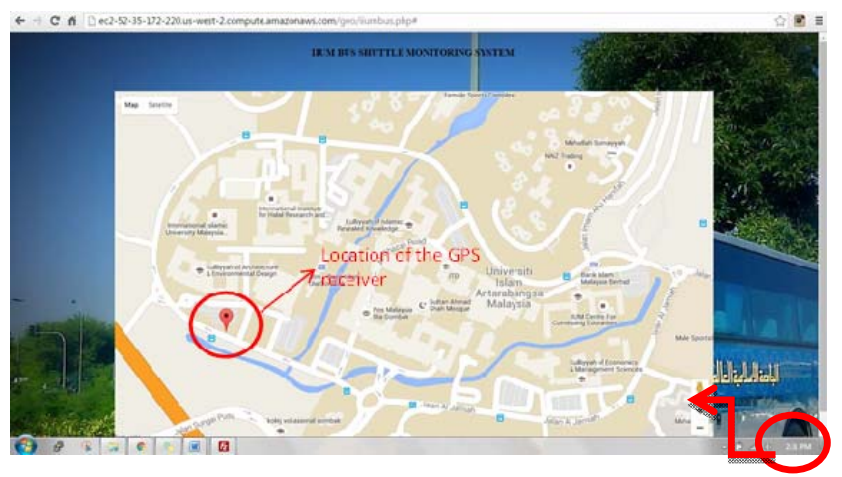

Figure 13. User map interface and location marking

The website can be accessed by opening the server domain 'http://ec2-52-35-172-220.us-west2.compute.amazonaws.com/geo/iiumbus.php\#'. The website shows IIUM campus road map on the Google Maps. A pointer indicates the current location of the GPS receiver.

GPS receiver is placed inside the IIUM bus, at the dashboard. The test has been conducted at 5 checkpoints from the total of 9 checkpoint bus stop in IIUM campus (Fig. 14). The GPS receiver placed in the bus was moving from one checkpoint to the other checkpoint to test the real time ability to get the location data.

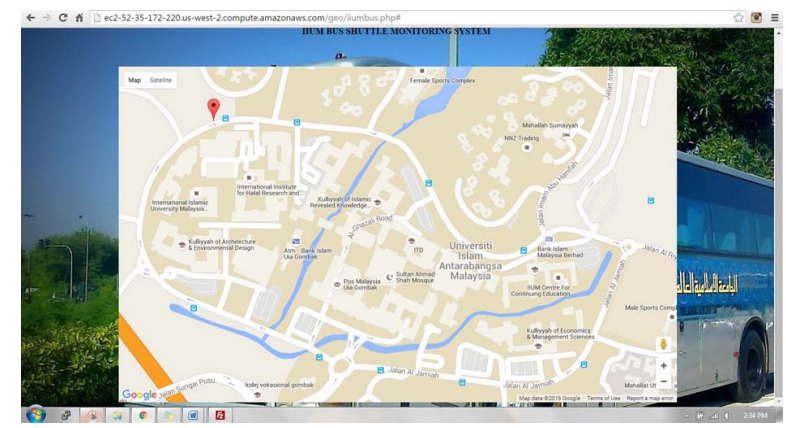

Figure 14. Location of GPS receiver (bus) at Kuliyyah Engineering bus stop

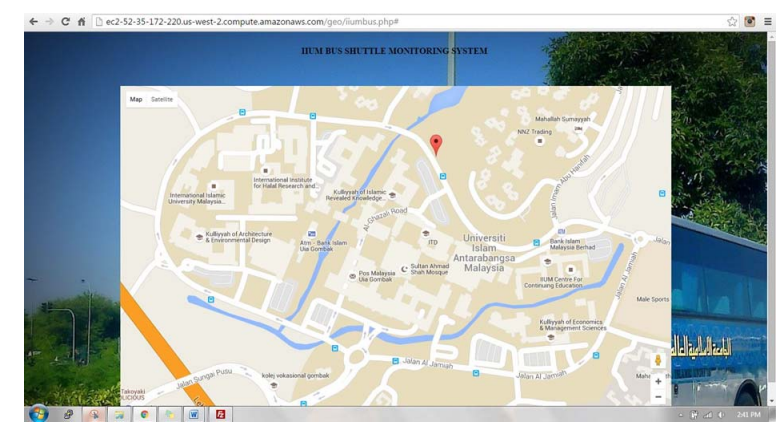

Figure 15. Location of GPS receiver (bus) at Mahallah Nusaibah bus stop

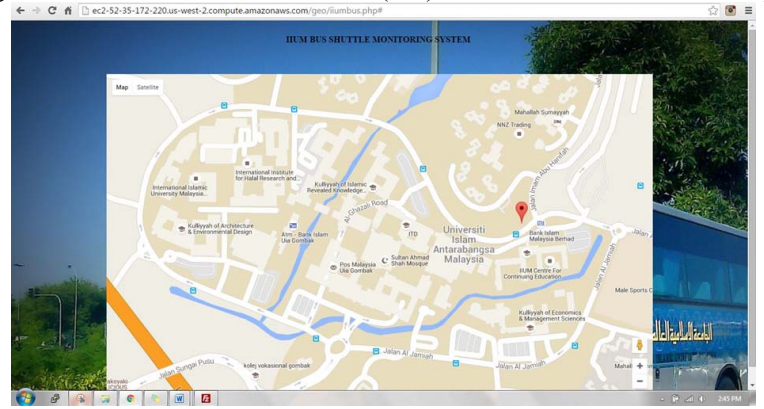

Figure 16. Location of GPS receiver (bus) at Kuliyyah of Law bus stop

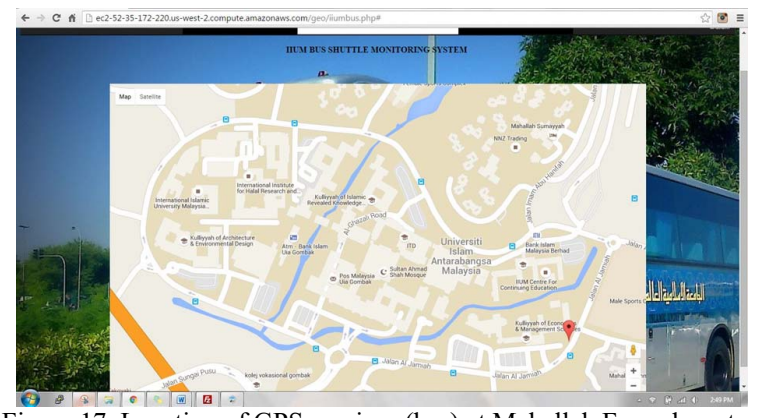

Figure 17. Location of GPS receiver (bas) at Mahallah Faruq bus stop

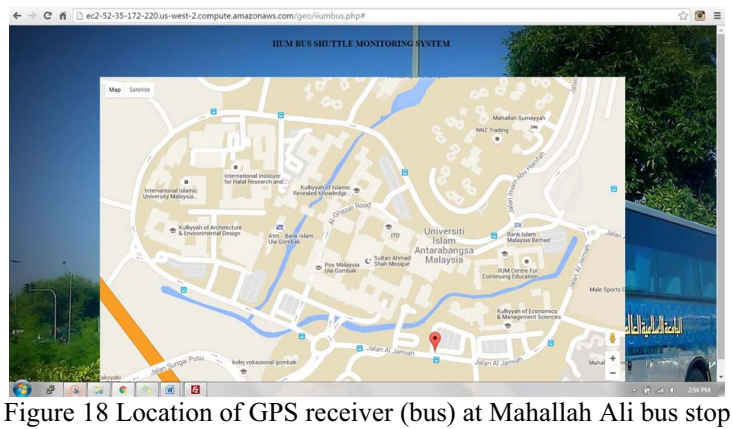

Fig. 15 to Fig. 18 show the results of real time tracking of the GPS receiver (bus). The pin pointer indicates the current location of the GPS receiver where the GPS receiver sends the data to the Ubuntu server. Exact time stamp is also shown in the figures. When the server receives the GPS data, a pin pointer will be shown on the road map of IIUM. 


\section{CONCLUSION}

The objective of this project, to develop a low cost realtime IIUM bus on campus tracking system has been successfully achieved. From the website, people can know the exact location of the bus in the real-time manner. More accurate GPS and better network connection can be considered for future improvement. Some suggestions for future improvements are:

- Increase speed of processor of the server. Free package service usually provides lower speed processor where data cannot be received and displayed smoothly.

- Use high gain GPS Antenna with better frequency range. Better signal transmission and faster GPS connections can be achieved.

- Improve Internet Network Connection by using 3G networks. In this project, GPRS was used as the internet carrier to send the data from the GPS receiver to the server. With the limitation of internet connection speed range about 56 to $114 \mathrm{kbps}$, sending data from GPS receiver to the server will a bit slow. Compare to $3 \mathrm{G}$ networks that reach speed up to $384 \mathrm{kbps}$ which is faster to accessing and sending data to the server.

\section{REFERENCES}

[1] Mohd Zuki W, "GSM/GPS vehicle tracking systemGtrack", M.S Thesis Information Technology) Faculty of Computer Science, Universiti Teknologi MARA, Shah Alam, Malaysia, April 2004, pp. 27-35.
[2] Lu, P. W., and Chen, R., "The dedicated short-range vehicle tracking", IEEE Vehicular Technology Conference, (6), 2010, pp. 1-5.

[3] Jesus Zegarra Flores and René Farcy, "GPS and IMU (inertial measurement unit) as a navigation system for the visually impaired in cities", Journal of Assistive Technologies, Vol. 7 (1), 2013, pp.47 - 56.

[4] Mistary P. V. and Chile R. H., "Real-time vehicle tracking system based on ARM7 GPS and GSM technology", IEEE India Conference, 2015, pp.1-6.

[5] Rycroft, M. J., "Understanding GPS Principles and applications", Journal of Atmospheric and SolarTerrestrial Physics, 1997.

[6] Garmin Inc, "An introduction to using a Garmin GPS", October 2005, Retrieved from http://www.garmin.com

[7] James B. Lingan, "Web server", 2015, Retrieved from www.whatis.techtarget.com

[8] Amazon, "Amazon Elastic Compute Cloud (Amazon EC2)", 2011, Retrieved from http://aws.amazon.com/ec2/

[9] Frederic Lardinois, "Google launches new maps APIs for location tracking and GPS-Free geolocation”, 2012, Retrieved from www.techcrunch.com

[10] Badamasi, Y. A. and Nigerian T. N. U., "The working principle of an Arduino", International Conference on Electronics, Computer and Computation (ICECCO), 2014, pp. 1-4. 